Revue Revue de l'histoire des religions

de Ihistoire des religions

Vision que vit Isaïe. Traduction du texte du prophète Isaïe selon la Septante de Alain LE BOULLUEC et Philippe LE MOIGNE, Index littéraire des noms propres et glossaire de Philippe LE MOIGNE Paris, Les Éditions du Cerf (« La Bible d'Alexandrie »), 2014

\title{
OpenEdition
} Journals

Édition électronique

URL : http://journals.openedition.org/rhr/8583

DOI : $10.4000 /$ rhr.8583

ISSN : 2105-2573

Éditeur

Armand Colin

Édition imprimée

Date de publication : 1 septembre 2016

Pagination : 420-423

ISBN : 978-2-200-93061-5

ISSN : 0035-1423

Référence électronique

Anne-Catherine Baudoin, «Vision que vit Isaie. Traduction du texte du prophète Isaïe selon la Septante de Alain LE BOULlUEC et Philippe LE MOIGNE, Index littéraire des noms propres et glossaire de Philippe LE MoIGNE ", Revue de l'histoire des religions [En ligne], 3 | 2016, mis en ligne le 06 octobre 2016, consulté le 23 septembre 2020. URL : http://journals.openedition.org/rhr/8583; DOI : https://doi.org/10.4000/rhr. 8583

Ce document a été généré automatiquement le 23 septembre 2020

Tous droits réservés 


\section{Vision que vit Isaïe. Traduction du texte du prophète Isaïe selon la Septante de Alain LE BOULLUEC et Philippe LE MOIGNE, Index littéraire des noms propres et glossaire de Philippe LE MOIGNE}

Paris, Les Éditions du Cerf (« La Bible d'Alexandrie »), 2014

Anne-Catherine Baudoin

\section{RÉFÉRENCE}

Vision que vit Isaïe. Traduction du texte du prophète Isaïe selon la Septante de Alain LE BOULLUEC et Philippe LE MOIGNE, Index littéraire des noms propres et glossaire de Philippe LE MOIGNE, Paris, Les Éditions du Cerf (« La Bible d'Alexandrie »), 2014, 368 p., 20 cm, $30 €$, ISBN 978-2-204-10308-4.

À la découverte de ce nouveau volume de la collection de la «Bible d'Alexandrie », deux éléments surprennent d'emblée, en bien. La couverture est blanche, et non bleue comme d'habitude. Il ne s'agit pas en effet du volume traditionnel comprenant l'introduction présentant le livre biblique, la traduction et l'annotation continue mêlant remarques philologiques, écarts avec le texte massorétique et interprétations anciennes du texte, mais de la traduction du texte grec d'Isaïe, préparée pour ce projet (p.170). Autre surprise, le titre, à la fois explicite et inhabituel, reprend les premiers mots du texte, dans une tradition plus juive que grecque ; peut-être ce choix soulignet-il d'une manière à la fois poétique et pédagogique que c'est bien à l'intention des Juifs que le texte d'Isaïe a été traduit en grec. 
2 Le lecteur est ainsi invité à découvrir en français la traduction grecque d'un texte hébreu, produite par le judaïsme alexandrin au II $^{e}$ siècle avant notre ère. Le souci de donner un accès immédiat au texte est marqué par la structure du volume, qui s'ouvre sur la traduction des soixante-six chapitres d'Isaïe (p.9-145), et se poursuit avec une étude présentant le texte et la traduction proposée (p.149-175), puis avec différents instruments éclairant la compréhension du texte (p.178-360, soit la moitié du volume) et permettant le maniement de l'ouvrage (p. 361-367).

3 La langue de la traduction est belle et précise, sauf à faire usage de mots relativement rares, et rend la lecture tout à fait agréable ; l'obscurité de certains passages n'est pas à imputer aux traducteurs français. La présentation avec deux niveaux de sous-titres prodigue le minimum d'apparat pour la fréquentation d'un texte à la fois familier et inconnu sous cette lumière. Qu'il me soit permis de poser juste quelques questions. Is 33, 17: certes le passage est peu clair et bathusphônos est un hapax (signalé par Lee p. 142, cf. infra), mais pourquoi choisir de rendre bathus par "confus»? Is 60, 4: puisque le grec utilise le pluriel, pourquoi ne pas traduire de manière peut-être plus habituelle «tes filles seront portées sur les épaules » plutôt que «sur l'épaule »? Enfin, j'ai beaucoup apprécié la traduction «le dessein qu'il a décidé » $(7,5 ; 8,10$ [avec «dont » comme relatif]; 14,$26 ; 19,17)$ qui rend d'une certaine manière le jeu étymologique hè boulè hèn ebouleusato. Pourquoi ne pas l'avoir adoptée aussi en 3, 9 ? Quant à la traduction de la formule en 31,6, qui met l'accent sur l'épithète batheian accolée à boulèn, "vous qui enfouissez votre dessein transgresseur " (littéralement: " vous qui décidez un dessein profond et transgresseur »), elle est belle mais lointaine.

4 L'étude qui suit, œuvre commune là encore d'Alain Le Boulluec et de Philippe Le Moigne, donne un avant-goût de ce que pourra être l'introduction au «volume bleu » d'Isaïe. Les quelques termes cités en grec pour les besoins de la démonstration sont translittérés, dans un souci de faciliter la lecture. L'étude s'ouvre avec la comparaison de quatre versets du texte LXX d'Isaïe et du texte hébreu qui montrent des divergences profondes; celles-ci ne reposeraient pas sur un écart entre le texte-source de l'Isaïe grec et le texte massorétique auquel nous avons accès aujourd'hui mais seraient dues à la théologie de l'auteur juif hellénophone, soucieux d'actualiser le message biblique - ce qui fait de lui un témoin de l'interprétation du texte. Grâce à différents indices, on pourrait dater la traduction de la fin de la décennie 140 avant notre ère, par un juif égyptien. Pour préciser de quel milieu serait issu ce traducteur, deux hypothèses, peutêtre compatibles, sont présentées : Léontopolis et ses prêtres fidèles à Onias III, ou les philologues d'Alexandrie. Les auteurs proposent ensuite quelques remarques sur leur traduction qu'ils ont voulue à la fois fluide à la lecture et fidèle à la coloration particulière du texte grec. Soulignons par exemple deux remarques: le choix de la traduction de doulos, " esclave ", par la périphrase «qui est à », explicitant à la fois la supériorité essentielle de Dieu sur son peuple choisi, mais aussi l'intimité du contact entre les deux (p.167-168); et le souci de refléter le texte dans son contexte juif hellénistique sans que la traduction se fasse déjà témoin de la réception chrétienne - à la différence du Commentaire sur Isaie de Théodoret de Cyr, ou simplement de Mt 1, 23, où parthenos est compris comme "vierge » dans la citation d'Is 7, 14 ("jeune femme " dans le texte proposé ici). On peut simplement regretter, par habitude philologique, que la mention de l'édition utilisée (Ziegler, dans la collection de Göttingen) n'apparaisse qu'à la page 169 , ce qui est sans doute dû, là encore, au désir de ne pas effaroucher le lecteur. 
Philippe Le Moigne a composé les deux répertoires qui suivent l'étude, un index littéraire des noms propres (p. 177-312) et un glossaire (p. 313-353). Le premier est constitué de notes utiles qui éclairent l'histoire, la géographie et la prosopographie. Le rassemblement des occurrences des termes dans le livre d'Isaïe ou dans le texte biblique et la synthèse proposée font de cet index un outil précieux, au-delà du support de lecture. Il est fait pour la lecture autant que pour la consultation : on n'aurait peutêtre pas pensé à y chercher les mots "Grèce », «Israël » ou "Étrangers ». Ce dernier mot pose la question des «noms propres", repérés par leur majuscule en français: ainsi, petra pourra-t-il être lu comme le nom commun « roche » ou comme le nom d'une ville édomite. Pour l'explication sur le nom grec d'Abraam sans aspiration en milieu de mot, il faudrait préciser " pas le moyen de noter dans la graphie une aspiration suivant une voyelle à l'intérieur d'un mot ». La graphie du nom de son épouse Sarra aurait pu aussi être expliquée: elle reflète l'orthographe grecque, alors que le nom de Saül traduit plutôt qu'il ne translittère Saoul. Il ne s'agit là que de détails - de brimborions, selon le terme de Le Moigne (p. 324).

6 Le glossaire français donne le sens des mots rares - dans mon ignorance j'y ai appris qu'un artabe (il aurait été utile de préciser le genre) est une unité de capacité et le hallier un domaine boisé - ou précise leur sens dans le contexte (catin, fleuve, îles - qui ne sont pas toujours des étendues de terre entourées d'eau). Là encore, on comprend que le terme grec, voire le mot hébreu, ne soient pas mentionnés même en translittération, mais certains lecteurs auraient pu trouver cela utile; de même, le désir de ne pas entraver la lecture a conduit à ne pas signaler dans le texte les mots qui apparaissaient dans l'index.

7 À la bibliographie présentée avec un grand souci de clarté (ouvrages utilisés, renvoi aux répertoires contenant des informations détaillées sur Isaïe selon la Septante, complétés par les références les plus récentes) pourra être ajouté l'article récent de John A.L. Lee, "The literary Greek of Septuagint Isaiah », Semitica et Classica 7 (2014), p. 135-146, qui reprend notamment un exposé donné à Paris dans le cadre des conférences sur la Bible grecque des Septante en avril 2013 et étudie le niveau de langue du texte d'Isaïe par rapport à d'autres textes de la Septante et de la littérature grecque.

8 Cet ouvrage à couverture blanche rejoindra utilement les ouvrages à couverture bleue, même après la parution à venir de l'Isaïe à couverture bleue : le travail présenté ici s'inscrit dans une perspective originale, où sont mis en avant le confort de lecture et la fluidité sans que cela porte préjudice à l'acribie et à la science. L'historien des religions peut être soulagé de disposer enfin de la traduction française d'un texte grec certes beau mais souvent difficile et obscur, et de trouver dans la brève étude et dans les riches index un soutien utile à l'exploration du texte. 


\section{AUTEURS}

\section{ANNE-CATHERINE BAUDOIN}

École normale supérieure, Paris. 